\title{
Canadian Consensus Conference: The FDA decision on the use of 5 ARls
}

\author{
Laurence Klotz, MD, FRCSC,* Michael Chetner, MD, FRCSC, ${ }_{;}^{+}$Joseph Chin, MD, FRCSC,; Tony Finelli, MD, \\ FRCSC;: Neil Fleshner, MD, FRCSC,; Yves Fradet, MD, FRCSC; $;$ Larry Goldenberg, MD, FRCSC,";

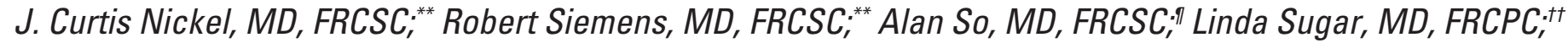 \\ Alexandre Zlotta, MD, FRCSC,; Eric Klein, MD,;土 Howard Parnes, MD,; David Penson, MD
}

\begin{abstract}
*Department of Urology, Sunnybrook Health Science Centre, University of Toronto, Toronto, ON; 'Division of Urology, University of Alberta Hospital, Edmonton, AB; \&Division of Urologic Surgery, Western

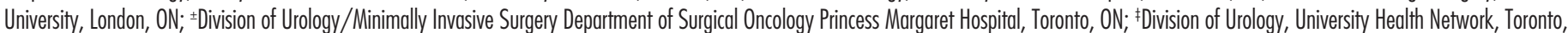
ON; * Department of Urology, Laval University, Quebec, QC; "Department of Urologic Sciences, UBC, Vancouver, BC; ${ }^{*}$ Department of Urology, Queen's University, Kingston, ON; ${ }^{+1}$ Department of Pathology, Sunnybrook Health Science Centre, Toronto, ON; \pm \pm Glickman Urological and Kidney Institute, Chairman, Glickman Urological and Kidney Institute, Cleveland, OH; 挂Prostate and Urologic Cancer Research Group, Division of Cancer Prevention, National Cancer Institute, Bethesda, MD; *Fivision of Urology, Vanderbilt University Medical Center; Nashville, TN
\end{abstract}

Cite as: Can Urol Assoc J 2012;6(2):83-8. http://dx.doi.org/10.5489/cuaj.12058

\section{Introduction}

In late 2010, the U.S. Food and Drug Administration's (FDA) Oncologic Drug Advisory Committee (ODAC) recommended against prostate cancer chemoprevention labelling for the 5-alpha reductase inhibitors (5ARIs). The ODAC met on December 1, 2010 to hear presentations from GlaxoSmithKline (GSK) and Dr. Ian Thompson (Merck) regarding dutasteride and finasteride. ${ }^{1}$ GSK was seeking a prostate cancer risk reduction label. Merck was not seeking a risk-reduction label, but rather a change in their product monograph. The FDA presented new, unpublished analyses of the data from the Prostate Cancer Prevention Trial (PCPT) and the REduction by DUtasteride of prostate Cancer Events (REDUCE) trial, as well as risk-benefit analyses unadjusted for detection-bias. ${ }^{2-4}$ The FDA asked the voting panel to consider whether the "real world" risk-benefit ratio was favourable for:

a) Finasteride in men $>55$ years old with a normal digital rectal examination (DRE) and prostate-specific antigen (PSA) $<3 \mathrm{ng} / \mathrm{mL}$

b) Dutasteride in men with an elevated PSA and a negative biopsy

In discussing the real world risks and benefits of these drugs, panel members expressed concern that some men would take the drug without adequate follow-up.

ODAC voted against recommending dutasteride for the prostate cancer risk reduction indication because, in the view of the ODAC members, the risk for an increase in highgrade tumours outweighed the benefits of prostate cancer risk reduction, given the potential for widespread use of this agent in the United States. The ODAC recommended against prostate cancer chemoprevention labelling for 5ARIs (Table 1).

\begin{tabular}{lccc}
\hline \multicolumn{4}{l}{ Table 1. Results of the ODAC vote chemoprevention } \\
\hline Vote & Opposed & In favour & Abstain \\
\hline Dutasteride & 14 & 2 & 2 \\
Finasteride & 17 & 0 & 1 \\
\hline
\end{tabular}

On June 9, 2011, the FDA notified health care professionals that the Warnings and Precautions section of the labels for 5ARIs was revised to include new safety information about the increased risk of high-grade prostate cancer. This risk appears to be low, but health care professionals should be aware of this safety information, and weigh the known benefits against the potential risks when deciding to start or continue treatment with 5ARIs in the approved indication for benign prostatic hyperplasia (BPH).

To review the FDA's decision from a Canadian perspective, the Canadian Urological Association (CUA), with direction from Dr. Laurence Klotz, assembled a team of experts and a meeting was convened on November 20, 2011 in Toronto, Ontario. The objectives of the meeting were as follows:

- To review the FDA's decision regarding the use of $5 \mathrm{ARI}$ in prostate cancer prevention, specifically with respect to the increase in high-grade cancer.

- To develop a Canadian consensus statement based on expert opinion and review of the evidence, on the use of 5ARIs in prostate cancer prevention and $\mathrm{BPH}^{3-9}$

The deliverables proposed by the consensus panel chair and the CUA Office of Education are as follows: (a) To prepare a Canadian statement on the use of 5ARIs in prostate cancer prevention, which reflects a broad consensus of 
Klotz et al.

academic and community practitioners, and primary care physicians with an interest in prostate cancer; (b) To publish this statement as a peer-reviewed article in CUA); and (c) To produce a patient brochure, which reflects this Canadian consensus.

Meeting participants were provided with all pertinent data, a description outlining the meeting objectives, expected outcomes and presentations and three position statements for voting (Appendices 1-4). After an introduction and review of the positions by Dr. Laurence Klotz, participants were asked to vote on the three positions prior to the initiation of discussion. After the initial vote, 7 presentations were made to the group:

1) A summary of the ODAC hearing and the FDA position: Laurence Klotz and David Penson

2) Personal take on the ODAC hearing as a participant: Howard Parnes

3) Pathology issues/Gleason scoring system: Linda Sugar

4) The CCO position on risk reduction of prostate cancer: Neil Fleshner

5) The modelling of cytoreduction and PSA effects: Eric Klein

6) The link between the FDA decision and the United States Preventive Services Task Force (USPSTF) screening decision: Laurence Klotz

7) Implications for use of 5ARIs in surveillance: Tony Finelli

\section{Key findings}

\section{Preliminary vote}

Attendees were given a ballot containing three positions (Appendix 1-4) and were asked to vote secretly.

- Position 1: The FDA Position (3 votes)

- Position 2: The "Pro" Position (3 votes)

- Position 3: The Middle Ground (6 votes)

\section{Expert Presentations}

\section{a) David Penson: A summary of the ODAC hearing}

Dr. Penson reviewed the ODAC decision and the data that were presented. His recommended use of 5ARIs moving forward is:

- Continue to use 5ARIs for BPH with the following proviso:

- Explain possible increased risk of high-grade prostate cancer to patients and DOCUMENT in chart - Closely monitor PSA kinetics after starting therapy
- Unclear if you need to send a letter to your patients currently on $5 \mathrm{ARIS}$

- Only consider 5ARI use for chemoprevention for men at increased risk of prostate cancer who are motivated to pursue chemoprevention

- Explain to patient that it is off-label use and highlight the possible risks of treatment and DOCUMENT in chart

- Closely monitor PSA after starting therapy and contact patient if he misses follow-up PSA mean scores or appointments to reschedule

\section{b) Howard Parnes: A personal take on the ODAC hearing as a participant}

Dr. Parnes also provided a summary of the decision-making process by the ODAC panel. Of note:

- $\quad$ ODAC met on 12/1/2010 to hear presentations by GSK and Merck regarding dutasteride and finasteride, respectively.

a. GSK was seeking a risk-reduction label

b. Merck was not seeking a risk-reduction label

- Merck stated that the post-hoc analyses addressing the observed increase in high-grade prostate cancer "did not rise to the level of a label."

- ODAC took the position that mortality reduction is the goal of chemoprevention

- Burden of prostate cancer was not considered by ODAC

- No weight was given to the possible role of detectionbias

- $\quad$ The addition of the "real world" setting did not leave much choice for the panel to vote in favour of dutasteride as widespread use has significant public health implications.

The crux of the controversy is whether the observed increase in high-grade cancer in the two trials was an artifact caused by several unavoidable biases associated with the use of 5ARIs, or represents a true increased risk. If the latter, it is unclear what the mechanism for the increase in high grade cancer is.

\section{c) Linda Sugar: Pathology issues/Gleason scoring system}

Dr. Sugar presented on pathology issues regarding modified Gleason score grading of needle biopsies. ${ }^{10}$ Of note:

- Even among expert genitourinary pathologists, there is interobserver variability in the reporting of the Gleason score.

The modified Gleason grading system has generally resulted in upgrading of the Gleason score, most frequently from 6 to 7 but also from 6 or 7 to 8 .

- In the original Gleason grading system, malignant glands with a cribriform pattern were assigned 
Gleason grade 3 . In the new grading system, most, if not all cribriform cancers are now designated Gleason grade 4 . With this narrowing of the definition of Gleason grade 3 and expansion of Gleason grade 4 , there are increased numbers of Gleason score $7(3+4)$ and $8(4+4)$ cancers. Another reason for the increase in Gleason 8 cancers has been the incorporation of a Gleason 5 tertiary pattern into the score (e.g., 3+5). ${ }^{10}$

- A result of this upgrading that has been an expansion of tumours that fall into the high-grade category (Gleason score 8-10).

- $\quad$ There is a need for longer term studies to assess the revised system and impact on long-term outcomes.

\section{d) Neil Fleshner: The CCO position on risk reduction of prostate cancer}

Dr. Fleshner reviewed a draft of the Cancer Care Ontario's guidelines on risk reduction of prostate cancer with respect to 5ARIs.

\section{e) Eric Klein: The modelling of cytoreduction and PSA effects}

Dr. Klein presented his opinion that it is reasonable to use $5 \mathrm{ARI}$ in patients at high risk for prostate cancer (tilting the cost benefit ratio towards the benefit side). He noted that it is important to focus discussion on identifying men at highest risk who are most likely to benefit. He defined high risk as: Elevated PSA (PSA above the population mean for men in their 40s), negative biopsy, family history and race. He indicated it is also important to refrain from implementing preventing therapy with 5 ARIs in low-risk patients (i.e., with low PSA $[<1.5 \mathrm{ng} / \mathrm{mL}]$ and no risk factors).

\section{f) Laurence Klotz: The link between the FDA decision and the USPSTF screening decision}

Dr. Klotz reviewed the USPSTF recommendation against PSA as a screening tool. ${ }^{11}$ The USPSTF determined that the risks of PSA screening outweigh the benefits due to over diagnosis and treatment of prostate cancer, particularly in men with low-grade disease who are unlikely to succumb to their disease. Noted by the consensus group:

1. The consensus conference delegates unanimously agreed that the use of PSA as a tool in case detection and as a follow-up tool for ongoing monitoring is where the emphasis should be. It was agreed that the mandate of this group was to develop Canadian recommendations on the use of 5ARIs, rather than question or debate the USPSTF recommendations.

2. The group agreed that the main benefit of 5ARIs for prevention was in populations who have PSA testing for early detection of prostate cancer. 5ARIs primarily reduce the risk of diagnosis of low-grade disease, which is largely screen detected. If widespread PSA testing is attenuated as a result of the USPSTF recommendation, this will reduce the benefit and role of 5 ARIs for prevention.

\section{g) Tony Finelli: Implications for use of 5 ARIs in surveillance}

Dr. Finelli presented preliminary data looking at men taking 5ARIs under active surveillance (at Princess Margaret Hospital). It was noted that fewer men required radical intervention while on 5ARIs and 5ARIs prevented pathologic progression of cancer.

\section{Consensus discussion}

The primary goal of the meeting was to discuss various positions regarding the place for 5ARIs in Canada in light of the December 2010 recommendations from ODAC. The following captures the key discussion points and areas of agreement from the meeting. This will serve as a preliminary table of contents for development of a position statement.

\section{Preamble/introduction}

- In the Canadian context, the panel believes that there is value in preventing low-grade cancers and decreasing the burden of prostate cancer.

- $\quad$ The FDA statement in perspective: The panel agrees that patients on 5ARIs require ongoing follow up, including periodic PSA screening and informed interpretation of the result. Asymptomatic low-risk men are not candidates for chemoprevention therapy with 5ARIs.

- $\quad$ Rise in PSA while on 5ARIs should trigger further review for evaluation.

- This is a class effect of 5ARIs.

- Risk/benefit discussion and monitoring is needed in all patients on 5 ARIs.

- Indication for 5ARIs: 5ARIs are indicated for the treatment of symptomatic BPH in men with enlarged prostates. Some of the following suggested uses for 5 ARIs are off label, but may still be appropriate in selected patients.

\section{Recommendations for the use of 5ARls}

For patients in these groups, treatment with 5ARIs should be accompanied by:

- Informing the patient of risks and benefits of treatment

- Monitoring and follow up. Men on 5ARIs should have a PSA after 6 months of therapy, and at least annually afterwards. A sustained rise in PSA should result in consideration of a biopsy. 
Klotz et al.

\section{Use of 5ARIs in BPH - Consensus attained}

5ARIs can be safely (or replace with benefit/risk statement) used for the treatment of lower urinary tract symptoms (LUTS)/BPH in men with an enlarged prostate with appropriate monitoring (DRE/PSA).

\section{Patients who have had a negative prostate biopsy and elevated PSA and are at increased likelihood for prostate cancer diagnosis - Consensus attained}

These patients may be offered 5ARIs with the goal of reducing their chance of a future diagnosis of prostate cancer. Patients should be informed that this approach will reduce their likelihood of being diagnosed with low-grade cancer, and a slight increased risk of high-grade cancer cannot be excluded.

2. In selected patients who have not had a prostate biopsy but have high concern or risk for a future diagnosis of prostate cancer, 5ARIs may be considered - Consensus attained

\section{Role of 5ARIs in patients on active surveillance - Consensus attained}

Although investigational, there are data to support the role of 5ARIs in maintenance of active surveillance patients with prostate cancer.

\section{Discussion}

- After a minimum of 6 months on 5ARIs, men adhering to therapy should see a decline in PSA

- $\quad$ Refer to CUA position on screening
- Rationale of clinical situations and why 5ARIs are appropriate

- Current Controversies: Given the dramatic increase in prostate cancer diagnosis, and the concerns about overdiagnosis expressed by the USPSTF, how significant is family history for identifying men at increased risk for prostate cancer? Should death or metastasis from prostate cancer in family members replace diagnosis of prostate cancer as a significant risk factor?

- In men on a 5ARI, what PSA kinetics triggers should be used to determine the need for a biopsy?

\section{Conclusions and next steps}

It was recommended that an introductory editorial from the CUA discussing differences in Canadian and U.S. healthcare delivery may help put a CUAJ article in context (specifically use and benefits of PSA). A second vote was not taken, because the original three positions put forward were modified and no longer applicable in their original format. It was agreed that a summary of the key meeting discussion points would be distributed to the group for further review and discussion.

Competing interests: None declared.

This paper has been peer-reviewed.

\footnotetext{
Appendix 1. Voting positions: The basic facts (agreed upon from all three perspectives)

The effect of 5ARIs in the prevention of prostate cancer has been studied in two pivotal trials, PCPT and REDUCE. PCPT compared finasteride $5 \mathrm{mg} /$ day to placebo in normal men over 55 with PSA <3.0. REDUCE studied men with an elevated PSA and a prior negative biopsy, considered at higher risk for prostate cancer than the PCPT population, who were randomized between dutasteride $0.5 \mathrm{mg} / \mathrm{day}$ versus placebo. In both studies, the endpoint was a prostate biopsy, performed at 7 years in PCPT and at 2 and 4 years in REDUCE. In both studies, patients were offered a 'for cause' biopsy if they developed a further rise in PSA or prostate nodule. These studies showed the following findings:

- PCPT and REDUCE showed a reduction in the likelihood of prostate cancer diagnosis of $25 \%$ to $30 \%$

- This reduction was in Gleason 6 prostate cancer

- For men on 5ARIs, PSA performs better as a marker for prostate cancer detection

- Both studies showed a small increase in the rate of diagnosis of high-grade prostate cancer (Gleason 8-10). This was both an absolute and a relative increase ( 280 vs. 237 in PCPT, i.e., 43 more cases on the finasteride arm out of 4368 men biopsied, i.e., an increase of 1\%); and an increase from 19 to 29 cases in REDUCE, i.e., 10 more cases out of 3298 on dutasteride, an increase of $0.3 \%$. On re-analysis of the REDUCE cases using the modified Gleason criteria, the Gleason 8-10 cancers increased from 0.5 to $1.0 \%$, an absolute increase of $0.5 \%$.

5ARIs: 5-alpha reductase inhibitors; PCPT: Prostate Cancer Prevention Trial; REDUCE: REduction by DUtasteride of prostate Cancer Events; PSA: prostate-specific antigen.
} 


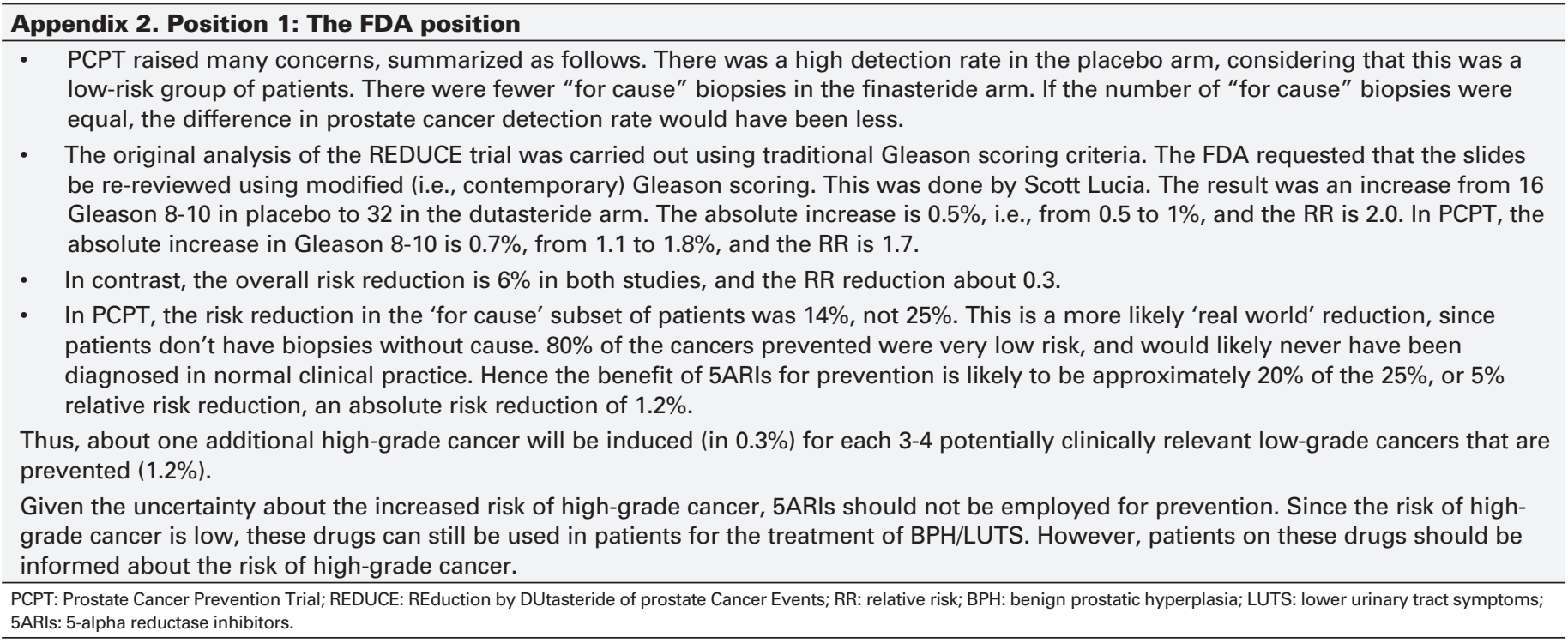

\section{Appendix 3. Position 2: The "Pro" position}

- The small increase in high-grade cancers is due to ascertainment bias introduced by the effect of 5ARls. This bias is due to two factors: gland cytoreduction and improved performance of the PSA.

- The re-analysis of pathology using the modified Gleason score in fact introduced two sources of variation from the original data: the effect of the modified Gleason scoring system, and the effect of inter-rater reliability. This was reflected in the reduced number of Gleason 7 tumors in the re-analysis. If 5ARIs increased high-grade cancer (i.e., Gleason pattern 4-5), one would have expected an increase in Gleason 7 disease.

- A number of modeling studies have sought to estimate the effect of gland cytoreduction and improved PSA performance. Correcting for ascertainment bias, these have estimated a reduction in all cancers of $39 \%$ and a reduction of high-grade cancer of $16 \%$. These studies were not accepted by the FDA. In a summary of the decision, Theoret calculated that, adjusting for change in prostate volume, the number of Gleason 8-10 cancers was increased by a factor of 1.5.

- This analysis assumes that volume reduction is uniform between peripheral zone and transition zone. In fact, many of these patients likely harboured anterior cancers. Recent data (not reviewed by the FDA) has shown that, amongst men with an elevated PSA and persistent negative biopsies, a substantial proportion harbour anterior cancers. These are often high grade (Margel et al, J Urol, in press). Anterior cancers in large prostates are notoriously difficult to target with TRUS guided biopsies. It is likely that the increase in high-grade cancer reflects nothing more than greater likelihood of detecting these cancers once the glands have been reduced in size by 5ARI treatment. In fact, the greater likelihood of finding high-grade anterior cancer may be a benefit of 5ARI.

- Further, the FDA discounted the benefit of avoiding a diagnosis of low-grade prostate cancer.

- Another source of imbalance in the REDUCE trial relates to the two sets of biopsies mandated, at year 2 and year 4 . There was no difference in high-grade cancer at the year two biopsy. However, during the first round of biopsies, 142 more patients had cancers detected in the placebo arm, likely because they grew more rapidly than those on Dutasteride. These patients were taken off study. Assuming that both groups had the same number of cancers at study entry, this means that 142 more patients in the Dutasteride group had cancers going in to the 2 nd round of biopsies at year 4 . Assuming that $8 \%$ of these cancers were in fact Gleason $8-10$, one would expect approximately 12 more Gleason 8-10 cancers identified in the Dutasteride group on the 2nd set of biopsies; exactly what was found ( 12 vs $1,0.5 \%$ vs $.04 \%$ ). Further, despite the Dutasteride group harbouring more cancers going into the 2 nd biopsy, and the improved performance of PSA in men on Dutasteride, the number of cases identified was significantly fewer.

- It is also likely that the men with high-grade cancer would have been identified and treated in a timely fashion. Using the National Comprehensive Cancer Network (NCCN) guideline for biopsy (NCCN Clinical Practice Guidelines, 2011, www.nccn.org), 3/4 of the highgrade cancer cases would have been diagnosed. There was no evidence that Dutasteride interfered with the detection of high-grade cancers using PSA.

- Economic analysis of the benefit of 5ARI in prevention suggests that the cost per cancer avoided is reasonable, and in line with other accepted prevention strategies.

- Therefore we believe the data from PCPT and REDUCE justifies the use of 5ARls in prostate cancer prevention. This is particularly true given the other benefits of the drug in the prevention of the sequelae of BPH progression.

5ARIs: 5-alpha reductase inhibitors; PSA: prostate-specific antigen; FDA: Food and Drug Administration; TRUS: transrectal ultrasound; REDUCE: REduction by DUtasteride of prostate Cancer Events; PCPT: Prostate Cancer Prevention Trial; RR: relative risk; BPH: benign prostatic hyperplasia. 
Klotz et al.

\begin{abstract}
Appendix 4. The middle ground
The effect of 5ARls in the prevention of prostate cancer has been studied in two pivotal trials, PCPT and REDUCE. PCPT compared finasteride $5 \mathrm{mg} /$ day to placebo in normal men $>$ age 55 with PSA $<3.0$. REDUCE studied men with an elevated PSA and a prior negative biopsy, considered at higher risk for prostate cancer than the PCPT population, who were randomized between dutasteride $0.5 \mathrm{mg} / \mathrm{day}$ vs placebo. In both studies, the end point was a prostate biopsy, performed at 7 years in PCPT and at 2 and 4 years in REDUCE. In both studies, patients were offered a 'for cause' biopsy if they developed a further rise in PSA or prostate nodule. These studies showed the following findings:

- PCPT and REDUCE showed a reduction in the likelihood of prostate cancer diagnosis of $25-30 \%$

- This reduction was in Gleason 6 prostate cancer

- For men on 5ARIs, PSA performs better as a marker for PCa detection

- Both studies showed a small increase in the rate of diagnosis of high-grade prostate cancer (Gleason 8-10). This was both an absolute and a relative increase ( 280 vs 237 in PCPT, i.e., 43 more cases on the finasteride arm out of 4368 men biopsied, i.e., an increase of $1 \%$ ); and an increase from 19 to 29 cases in REDUCE, i.e., 10 more cases out of 3298 on Avodart, an increase of $0.3 \%$. On reanalysis of the REDUCE cases using the modified Gleason criteria, the Gleason 8-10 cancers increased from 0.5 to $1.0 \%$, an absolute increase of $0.5 \%$. The observation of an increase in absolute numbers of high-grade cancers seen in both PCPT and REDUCE is consistent. It is likely that the reasons for this increase are multifactorial. As demonstrated in multiple modelling studies, the effects of cytoreduction of the gland and increased performance of PSA at identifying men with high-grade cancer biased the studies towards finding more high-grade cancer in the $5 A R I$ arms. These studies also suggest plausibly that the true effect of 5ARIs is a decrease in high-grade cancer. However, the possibility that, in rare patients, high-grade cancers may be induced cannot be dismissed completely. This effect is likely to occur in at most one in 200 patients, and likely in considerably fewer than this. Further, there are considerable benefits of avoiding a diagnosis of low-grade prostate cancer, including avoiding the 'survivor' label, and avoiding a risk of radical treatment. 5ARIs also have other benefits, in terms of reduced sequelae of BPH progression.

While it is not possible to exclude with certainty the possibility that a small increased risk of highgrade cancer exists associated with $5 A R I$ use, the preponderance of evidence suggests that most or all of the increased high-grade cancers seen in the two trials are related to ascertainment bias. Patients at high risk for prostate cancer thus benefit from the risk reduction associated with 5ARI treatment. Such patients should be counselled as to the risks and benefits of 5ARI treatment, including both the substantial reduction in the risk of diagnosis, and the slight increased risk of high-grade cancer. Such patients include those with a strong family history, racial predisposition, and persistently elevated PSA. The use of 5ARIs for men with BPH/LUTS should not be significantly influenced by this concern. Men who are not at increased risk for prostate cancer and have no BPH/LUTS but are concerned about reducing prostate cancer risk should be offered dietary and lifestyle modification (discontinue smoking, exercise, obesity avoidance, and dietary modification) rather than 5ARIs.

5ARIs: 5-alpha reductase inhibitors; PSA: prostate-specific antigen; REDUCE: REduction by DUtasteride of prostate Cancer Events; PCPT: Prostate Cancer Prevention Trial; RR: relative risk; BPH: benign prostatic hyperplasia; LUTS: lower urinary tract symptoms.
\end{abstract}

\section{References}

1. U.S. Food and Drug Administration. December 1, 2010: Oncologic Drugs Advisory Committee Meeting Announcement. Available online at: http://www.fda.gov/AdvisoryCommittees/Calendar/ucm230653. htm. Accessed on March 22, 2012.

2. Croker KS, Ryan A, Morzenti T, et al. Delivering prostate cancer prevention messages to the public: How the National Cancer Institute (NCI) effectively spread the word about prostate cancer prevention trial (PCPT) results. Urol Oncol 2004;22:369-76.

3. Andriole GL, Bostwick DG, Brawley OW, et al; REDUCE Study Group. Effect of dutasteride on the risk of prostate cancer. N Engl J Med 2010;362:1192-202.

4. Andriole GL, Bostwick DG, Brawley OW, et al; REDUCE Study Group. The effect of dutasteride on the usefulness of prostate specific antigen for the diagnosis of high grade and clinically relevant prostate cancer in men with a previous negative biopsy: results from the REDUCE study. J Urol 201 1;185:126-31.

5. Lotan Y, Cadeddu JA, Lee JJ, et al. Implications of the prostate cancer prevention trial: a decision analysis of survival outcomes. J Clin Oncol 2005;20:1911-20.
6. Theoret MR, Yang-Min N, Zhang JJ, et al. The risks and benefits of 5a-reductase inhibitors for prostatecancer prevention. N Engl J Med 2011;365:97-9.

7. Thompson IM, Pauler, Goodman PJ, et al. Prevalence of prostate cancer among men with a prostate-specific antigen level < or = $4.0 \mathrm{ng}$ per millimeter. N Engl J Med 2004;350:2239-46.

8. Thompson IM, Goodman PJ, Tanger $C M$, et al. The influence of finasteride on the development of prostate cancer. N Engl J Med 2003;349:215-24.

9. Unger J, Thompson IM, LeBlanc, et al. Estimated impact of the Prostate Cancer Prevention trial on population mortality. Cancer 2005;103:1375-80.

10. Epstein, Jonathan I, Allsbrook, et al. The 2005 International Society of Urological Pathology (ISUP) Consensus Conference on Gleason Grading of Prostatic Carcinoma. Am I Surg Pathol 2005;29:1228-42.

11. U.S. Preventive Services Task Force (UPSTF). Screening for prostate cancer: A review of the evidence for the U.S. Preventive Task Force. Available at: http://www.annals.org/content/early/2011/10/07/00034819-155-11-201112060-00375.1. fullabout:Tabs. Last accessed on March 22, 2012.

Correspondence: Dr. Laurence Klotz, Sunnybrook Health Sciences Centre, 2075 Bayview Ave., Room MG 204 Toronto, 0N M4N 3M5; fax: 416-480-6121; laurence.klotz@sunnybrook.ca 